\title{
Research on Optical System Focal Length Measurement Technology based on CCD Imaging Technology
}

\author{
Yao Qinghua $^{1}$ and Cao Miao ${ }^{2}$ \\ ${ }^{1}$ Changchun University Of Technology, Jilin Changchun, 130012, China, \\ ${ }^{2}$ Changchun University of Science and Technology, Jilin Changchun, 130022, \\ China \\ 188960880@qq.com, ${ }^{2} 79816540 @ q q . c o m$
}

\begin{abstract}
This paper puts forward a new method to measure focal length of optical system automatically and intelligently. This method can meet the quirements of fast, high-precision measurement, which integrated CCD imaging technology, grating angular displacement measuring technology and digital image processing technology. With the combined measurement method,the system combine the measured optical system with additional lens into the Galileo telescope system(the measurement optical system is the concave lens) and Kepler telescope system(the measurement optical system is convex lens).The parallel light, given by porro board and the collimator in the system, goes through the whole telescope system, and the focal length of the measured lens is calculated by measuring the magnification of the telescope system. The system ingeniously converts the focal length measurement to the angle measurement of off-axis light. In the process of measurement, the Fibonacci search algorithm is used to realize the automatic focus system, and the sub-pixel edge detection algorithm is implied to realize the double clamp line alignment. The measured results of the focal length of the lens group show that, the measurement speed is fast with the proposed method, and the uncertainty can be up to 10-4 orders of magnitude in this paper.
\end{abstract}

Keywords: Focal length measurement; Optical system; CCD; Error analysis

\section{Introduction}

In the telescope, microscope and a parallel light tube optical system, the lens or the eyepiece focal length is an important parameter of the optical system design. It is the basis of various optical system dimension and other optical parameters calculation and measurement [1-3]. The traditional methods of measuring the focal length of the lens are object distance image distance method, secondary focus method, auto collimation method and parallel light tube method. And these methods are all implied the location parameter into the focal length calculation of the measured lens in order to acquire a clear image.Therefore, the height and the accuracy of the measuring accuracy directly affect the quality and performance of the optical system. It is necessary to perform a new measurement with high precision of the focal length of the lens.

\section{Optical System Focal Length Measurement Design}

\subsection{System Components and Structural Design}

System composition principle diagram is shown in Figure 1. This system is mainly composed of xenon lamp, monochromator, linear guide, optical bench, axial Angle encoder, servo motor, CCD imaging system, optical system, a data processing system, etc. The radiation source is a xenon lamp with wide spectrum and high radiation intensity. 
The first stage optical system monochromator will modulation respectively Xenon lamp light source for line of d, e, F, C, g monochromatic light.Light illuminates the Porro plate which is placed on the focal plane of the lens. A pair of lines on the Porro plate are as the imaging target, which goes through the collimator collimating lens and modulates into parallel light, and then the lines go through pre-optical system and detected by the CCD imaging system. CCD searches reticles imaging angle on Porro plate with the servo motor rotation, and the measured lens focal length is obtained by numerical calculation.[4-6]

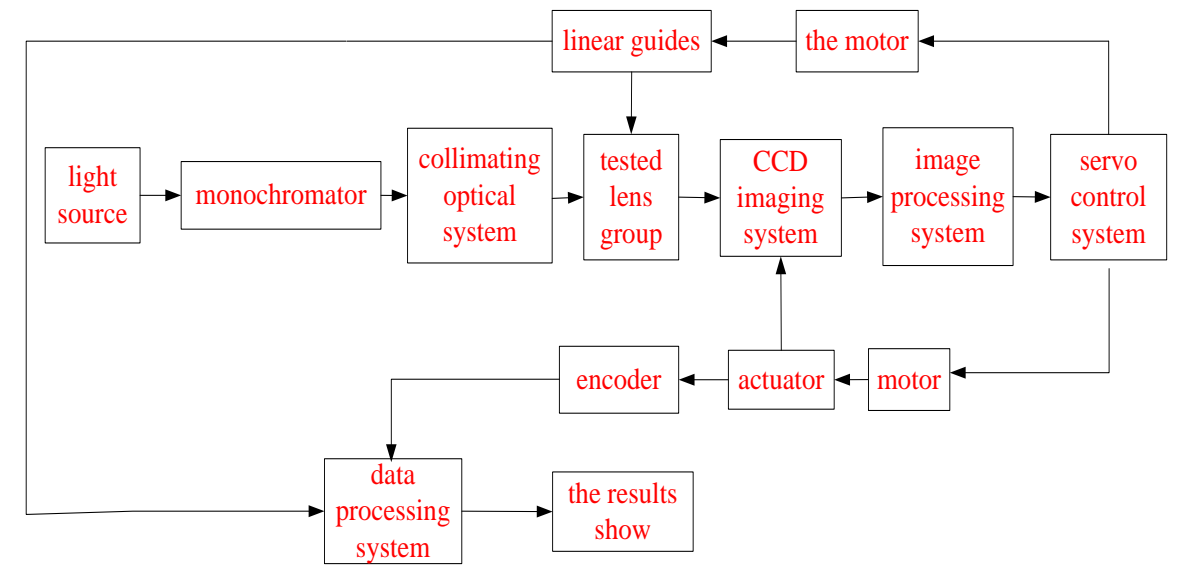

Figure 1. Block Diagram of System Components

Schematic diagram of system structure is shown in Figure 2.

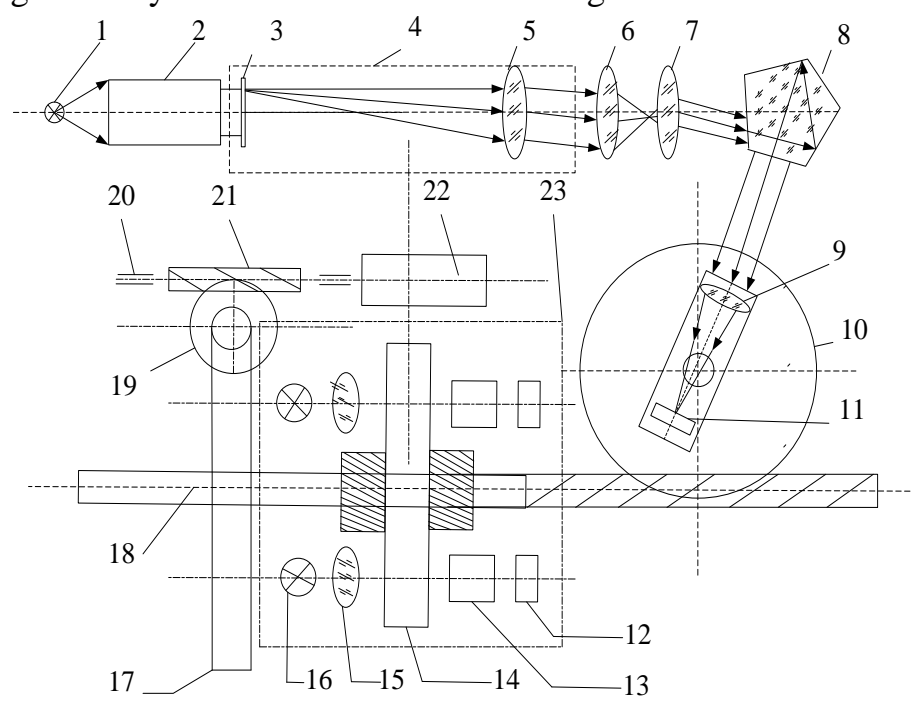

Figure 2. Schematic Diagram of System Structure

1-light source; 2-grating monochromator; 3- porro board; 4-parallel light pipe; 5- collimating lens; 6- additional lens; 7- measured lens; 8- pentaprism; 9- CCD imaging lens; 10-worm gear; 11- CCD detector; 12-photodiode; 13-indicating grating; 14-circular grating; 15lens; 16- light emitting diode; 17- turntable; 18- spindle; 19-worm gear; 20-support; 21-worm; 22- stepper motor; 23- encoder component

The full spectrum xenon lamp is used in this system to obtain the wavelength $656.3 \mathrm{~nm}, 486.1 \mathrm{~nm}, 587.6 \mathrm{~nm}, 435.8 \mathrm{~nm}, 546.1 \mathrm{~nm}$ of five different spectra monochromatic source of $\mathrm{F}, \mathrm{C}, \mathrm{d}, \mathrm{g}$ and e respectively. The spectral range of xenon lamp is $400 \mathrm{~nm}-700 \mathrm{~nm}$, and the spectral range is distributed continuously without wiring. So the lamp can ensure the measuring requirement for light source. The grating monochromator 
will modulate xenon lamp light to monochromatic spectrum required for measurements, which avoids the trouble of replacing lamp[7-9].

The collimating system is composed of parallel light pipe collimating lens. The incident monochromatic light will be collimated by the system, and formes a parallel monochromatic light. Light is parallel to the optical axis, and goes through pre-optical system and the measured lens. The parallel light is received by CCD imaging device.

The video signal uptaked by CCD will be converted into digital signal with image acquisition card. The digital signal can be recognized by computer and then transmitte image data to computer's memory real-time by the PCI bus. Finally, the real-time image is processed and displayed by image processing software, and the double groove images are obtained in parallel light pipe slits.

Before measuring deflection angle, In order to, the position of the measured lens must be adjusted to realize focusing alignment and ensure the CCD imaging clearly. By adjusting the stepper motor which mounted on an optical bench, the lens is derived far away or close to the CCD in order to achieve the image movement focusing and alignment.

The worm and gear are derived by the stepper motor, drive CCD to achieve the deflection angle search and targeting. When the preset double lines not clamped single line and need for alignment, the stepper motor derived by the software controls CCD rotation, locks target after aligning. After the photoelectric encoder measured deflection angle, the data uploaded to the PC are calculated and processed, finally the measured lens focal length is obtained.

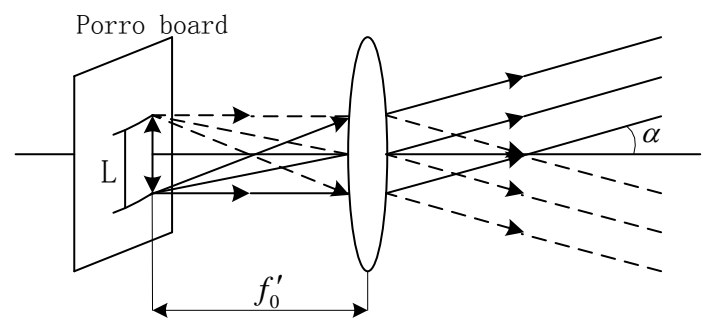

Figure 3. Schematic Diagram of Parallel Light Pipe

The porro plate engraved with double lines is located on the focal plane of the collimator lens, the lights through the collimator lens and the reticule become the parallel lights that have the angle with the optical axis:

$\tan \alpha=\frac{L}{2 f_{0}^{\prime}}$

In the above formula:

L-Porro plate distance between two scribed lines;

$f_{0}^{\prime}$ - the focal length of the collimator collimating lens;

$\alpha$-the angle between the collimator emitting light and the optical axis.

\subsection{Principle of Focal Length Measurement}

1.2.1. Convex Lens Focal Length Measurement: When the measured lens is a convex lens, the testing optical system is composed of additional lens and convex measured lens. The additional lens is convex lens, and the image focus coincidence with the object focus of the convex lens to be measured, so the afocal system-the Kepler telescope system is formed. Therefore, after off-axis parallel rays that have an angel $\alpha$ with the optical axis incident to the measured optical system. Emergent ray is off-axis parallel light as 
usual and has an $\beta$ angle with the optical axis. The optical measurment optical system of the convex lens group are shown in Figure 4.

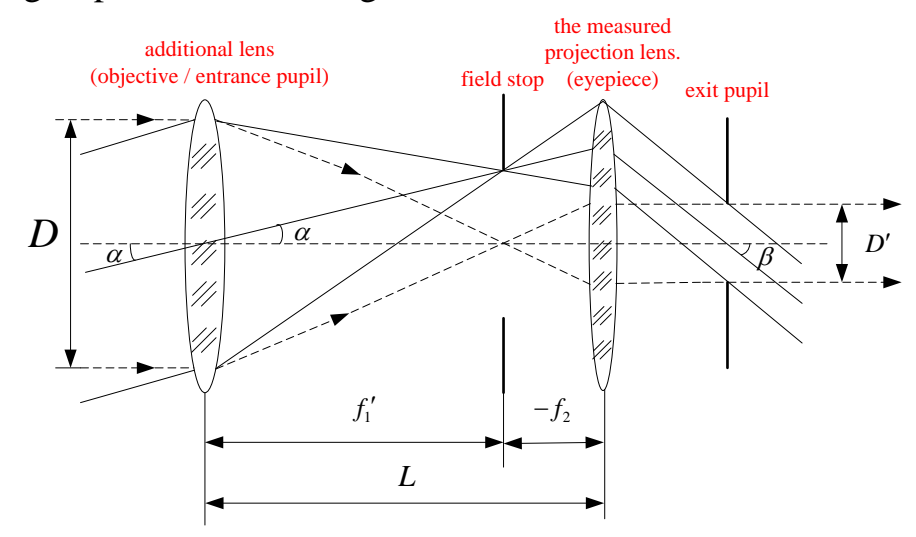

Figure 4. The Focal Length of the Convex Lens Group Testing Optical
System

As shown in Figure 4, the optical measurment optical system of the convex lens group is Kepler telescope system. The visual magnification is the ratio of the image of distant objects into the tangent of the angle to the eyes and the tangent angle the object directly to the eyes. Two edges parallel rays intersect at two lens focus coincidence according to the similar triangle theorem get in figure. The ratio is expressed by $\Gamma$.

$\Gamma=\frac{\tan \beta}{\tan \alpha}=-\frac{D}{D^{\prime}}=-\frac{f_{1}^{\prime}}{f_{2}^{\prime}}$

Simultaneous solution can be obtained by formula (1.1) and (1.2):

$f_{2}^{\prime}=-\frac{f_{1}^{\prime} L}{2 f_{0}^{\prime} \tan \beta}$

According to paraxial ray tracing equations:

$\left\{\begin{array}{l}n^{\prime} u^{\prime}=n u-y_{i} \phi \\ y_{i+1}=y_{i}+u^{\prime} \Delta\end{array}\right.$

In the above formula:

$n$ and $n^{\prime}$ are the object space refractive index of the medium and image refractive index of the medium respectively;

$u$ and $u^{\prime}$ are the aperture angle of incidence and the exit aperture angle respectively;

$\phi$ is the optical power, which is equal to the reciprocal of the focal length of the lens;

$y_{i}$ and $y_{i+1}$ are intersection height respectively that light in section $i$ and $i+1$ optical glass;

$\Delta$ is the optical spacing, which is the distance between two pieces of lens.

The principal ray goes through the lens center. Its y value is 0 , and aperture angle of incidence is $\alpha$. The whole system is in air, therefore $n_{\text {and }} n^{\prime}$ are all 1 . The interval between the two pieces of lens is the sum of their focal length, and the focal length of the additional lens and the test lens are respectively $f_{1}^{\prime}$ and $f_{2}^{\prime}$. The ray tracing equation after the principal ray through additional lens is: 
$\left\{\begin{array}{l}u^{\prime}=\alpha \\ y_{2}=u^{\prime}\left(f_{1}^{\prime}+f_{2}^{\prime}\right)\end{array}\right.$

Similarly, the ray tracing equation after the principal ray through a convex test lens is

$\beta=\alpha-\frac{\alpha\left(f_{1}^{\prime}+f_{2}^{\prime}\right)}{f_{2}^{\prime}}=-\alpha \frac{f_{1}^{\prime}}{f_{2}^{\prime}}$

Because the tracing rays is paraxial light, $\alpha$ and $\beta$ are all small, approximately:

$\left\{\begin{array}{l}\tan \alpha=\alpha \\ \tan \beta=\beta\end{array}\right.$

So formula (1.1), (1.6) and (1.7) are combined, it is available:

$f_{2}^{\prime}=-\frac{f_{1}^{\prime} L}{2 f_{0}^{\prime} \tan \beta}$

1.2.2. Concave Lens Group of Focal Length Measurement: When the measured lens is a concave lens, the testing optical system is composed of additional lens and concave measured lens. The additional lens is convex lens, and the image focus is coincidence with the object focus of the convex measured lens, so the composition of the afocal system-the Galileo telescope system. Therefore after off-axis parallel rays have an angel $\alpha$ with the optical axis incident to the measured optical system, and emergent rays are still off-axis parallel light. The angle with the optical axis is $\beta$, and the focal length of the concave lens group testing optical system is shown in figure 5.

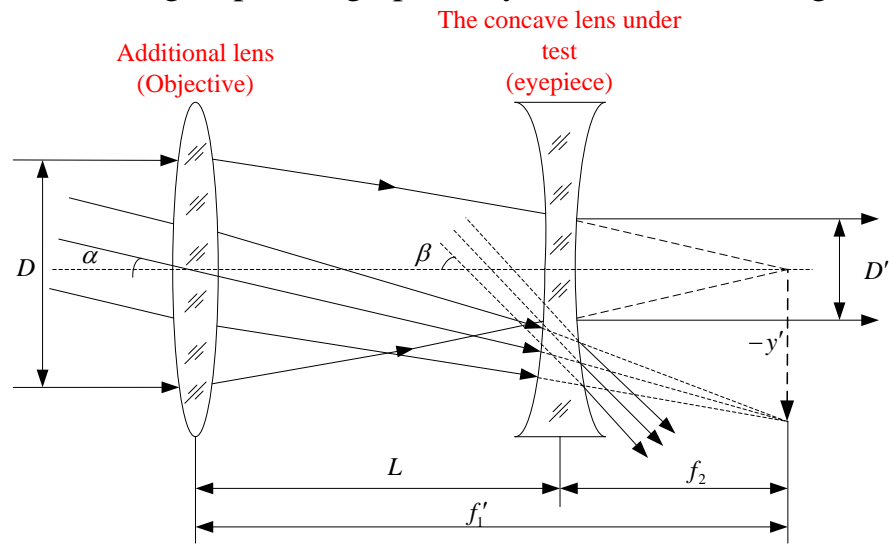

Figure 5. The Focal Length of the Concave Lens Group Testing Optical System

The focal length of the lens group test optical system is Galileo telescope system in Figure 5, and the visual magnification $\Gamma$ is:

$\Gamma=\frac{\tan \beta}{\tan \alpha}=\frac{D}{D^{\prime}}=\frac{f_{1}^{\prime}}{f_{2}^{\prime}}$

By formula (1.1) and (1.9), it can be obtained: 


$$
f_{2}^{\prime}=\frac{f_{1}^{\prime} L}{2 f_{0}^{\prime} \tan \beta}
$$

The ray tracing equation after the principal ray through additional lens is:

$$
\left\{\begin{array}{l}
u^{\prime}=\alpha \\
y_{2}=u^{\prime}\left(f_{1}^{\prime}-f_{2}^{\prime}\right)
\end{array}\right.
$$

The ray tracing equation after the principal ray through a convex measured lens is:

$$
\beta=\alpha+\frac{\alpha\left(f_{1}^{\prime}+f_{2}^{\prime}\right)}{f_{2}^{\prime}}=-\alpha \frac{f_{1}^{\prime}}{f_{2}^{\prime}}
$$

Because the tracing rays is paraxial light, $\alpha$ and $\beta$ are all small, approximately:

$$
\frac{\tan \beta}{\tan \alpha}=\frac{f_{1}^{\prime}}{f_{2}^{\prime}}
$$

So by formula (1.1) and (1.13), it can be obtained:

$$
f_{2}^{\prime}=\frac{f_{1}^{\prime} L}{2 f_{0}^{\prime} \tan \beta}
$$

\section{The CCD Imaging and Automatic Focus}

First, the motor can be controlled on parallel rails operation, the measured lens are driven to move around. That is auto focusing process to search for the best imaging position. The object focus of the measured lens is coincidence with image focus of the additional lens, and the light from the measured lens is parallel light. That can be received by CCD imaging system and make into a clear digital image, and it is the focusing alignment success. And then system controls the motor operation, drives the CCD imaging system, and search for the image make formed by the two lines on the Porro board. The rotation angle of CCD imaging system is recorded aligned to the two lines, which is the angle formed between the two tick lines. At last, to the focal length of measured lens is worked out by numerical method. The software flow chart is shown in Figure 6. 


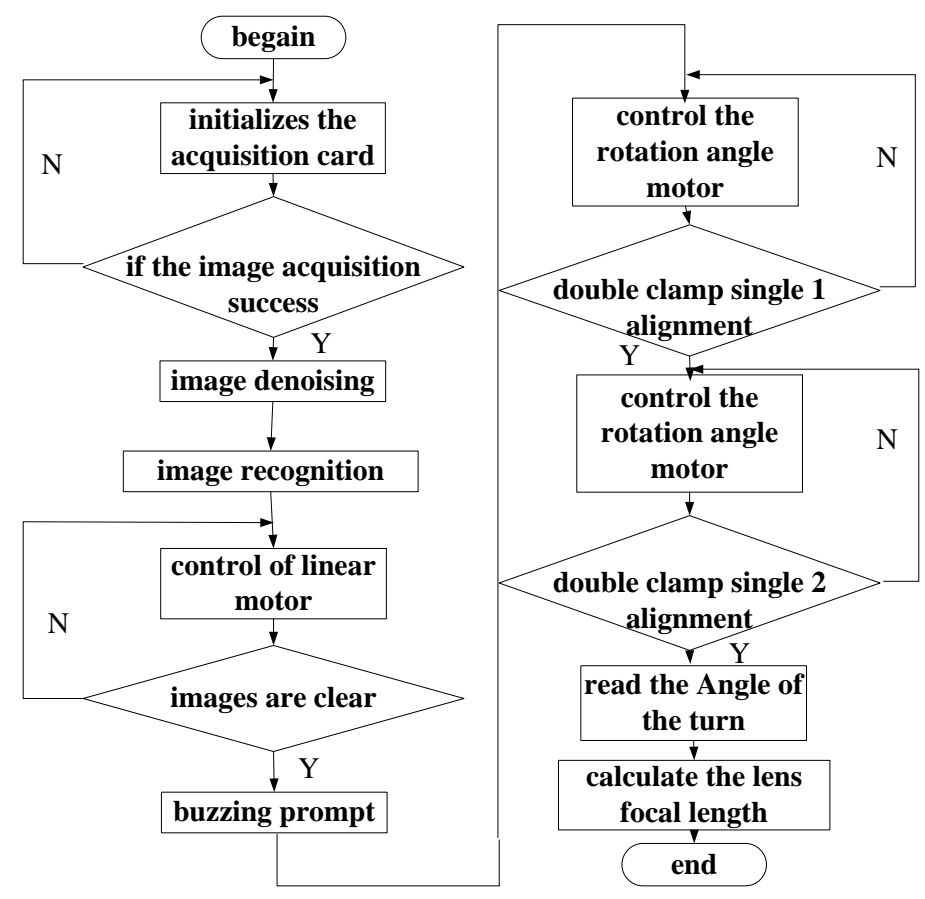

Figure 6. Focal Length Measurement Processes

Automatic focusing process includes image preprocess, the focusing window selection, focusing evaluation function values calculation and the motor search. In order to fast search the best focus position of the shot, namely the optimization of focusing search algorithm, the design of the system is based on Fibonacci focusing search algorithm. Fibonacci focusing search algorithm is a kind of optimal unimodal search algorithms of mathematical theory, it search unimodal curve pole by narrowing the interval range. This algorithm is based on one-dimensional search of the Fibonacci function realization, Fibonacci function expression is as follows:

$$
F(n)=\left\{\begin{array}{l}
F(n)=1, n=0,1 \\
F(n)=F(n-1)+F(n-2), n=2,3,4, \ldots
\end{array}\right.
$$

The principle of the Fibonacci search method is to assume the existence of extremal interval $[0, \mathrm{~b}]$, then take two points a1, a2 in the interval, and $a_{1}=t, a_{2}=b-t$ are as shown in Figure 7. Fibonacci function is $F(n) / F(n+1) \geq 0.5$ (take equal sign only $\mathrm{n}=1)$. Suppose that there is a maximal in interval $[0, \mathrm{~b}]$, when $f\left(a_{1}\right) \leq f\left(a_{2}\right)$, the next stop search interval is narrowed down to $\left[0, a_{1}\right]$; when $f\left(a_{1}\right)>f\left(a_{2}\right)$ conversely, the next stop search interval is narrowed down to $\left[\mathrm{a}_{2}, \mathrm{~b}\right]$. It repeat the process continue after the next search interval is determined. The Fibonacci function shows that search algorithm is one iteration, and search interval is reduced to $F(n) / F(n+1)$ of the last. The size of the search interval is into $\delta=b / F(n)$ after $n$ iterations. $\delta$ is the minimum precision after $n$ iterations, and then searching is stopped when $\delta$ is less than or equal to the accuracy of focusing. 

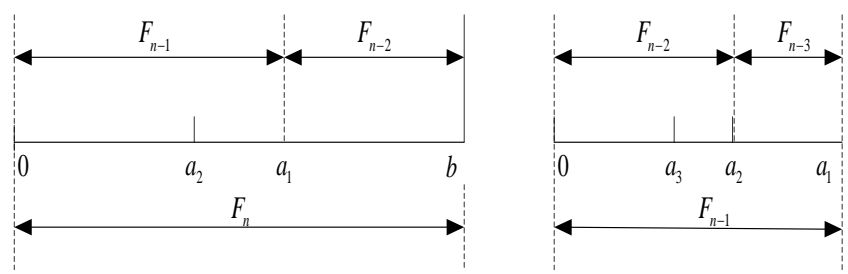

\section{Figure 7. Schematic Diagram of the Fibonacci Search Method}

Assuming that the focus position of the imaging system is located in the interval [a1, b1], and the focus position is corresponding to the maximum value of the focus evaluation function. In order to obtain the focusing accuracy $\varepsilon \cdot\left(b_{1}-a_{1}\right),(0<\varepsilon \leq 1)$, the steps of search focus position by using Fibonacci are as follow:

(1) Calculating the iteration times $n$ with $\varepsilon \cdot\left(b_{1}-a_{1}\right)$, assuming that

$$
\lambda_{k}=a_{k}+(F(n-2) / F(n)) \cdot\left(b_{k}-a_{k}\right), \mu_{k}=a_{k}+(F(n-1) / F(n)) \cdot\left(b_{k}-a_{k}\right)_{, \mathrm{k}=1 ;}
$$

(2) Comparison $f\left(\lambda_{k}\right)$ and $f\left(\mu_{k}\right)$, if $f\left(\lambda_{k}\right)>f\left(\mu_{k}\right)$, it skips to step (3); if $f\left(\lambda_{k}\right) \leq f\left(\mu_{k}\right)$, it skips to step (4);

(3) $a_{k+1}=a_{k}, b_{k+1}=b_{k}, \lambda_{k+1}=\mu_{k}, \mu_{k+1}=a_{k+1}+(F(n-k-1) / F(n-k)) \cdot\left(b_{k+1}-a_{k+1}\right)$, when $k=n-2$, it skips to step (5); otherwise, it jumps back to step (2);

(4) $a_{k+1}=a_{k}, b_{k+1}=b_{k}, \mu_{k+1}=\lambda_{k}, \lambda_{k+1}=a_{k+1}+(F(n-k-1) / F(n-k)) \cdot\left(b_{k+1}-a_{k+1}\right)$, when $k=n-2$,it skips to step (5); otherwise, it jumps back to step (2);

(5) $\lambda_{n}=\lambda_{n-1}, \mu_{n}=\lambda_{n-1}+\varepsilon$.If $f\left(\lambda_{n}\right)>f\left(\mu_{n}\right), a_{n}=\lambda_{n}, b_{n}=b_{n-1}$,otherwise $a_{n}=a_{n-1}, b_{n}=\mu_{n}$ and it skips to step (5);

(6) The system takes a point $t$ in the interval $\left[a_{n}, b_{n}\right]$, and outputs the value of ' $t$ ' as the focus position which satisfies the requirement of focusing precision. The search ends.

Fibonacci focusing search method is for determining the scope of the search and search precision, the sampling points that it need to search are fixed, and the motor trip is also fixed. This method has the advantages of high accuracy and shorter search time.

\section{Double Slit Clamp Single Alignment}

The image acquisition card used in this system is a black and white image acquisition card, and the image got through the image acquisition card is level 256gray image. The collected gray image are processed to achieve the machine vision automatic alignment, identify and analysis.

The key to using CCD target is scribed line coordinates accurately in the image processing method. Sub-pixel edge detection algorithm is used in this system to achieve single-lane alignment clip, and the basic steps are as follows:

(1) The traditional edge detection operator is used to coarse location the light deflection. The digital image of each pixel of the neighboring points' gray weight is studied differently, and the nearest neighboring points are weighted. The available operator is as follows:

$$
\begin{aligned}
s(i, j) & =\left|\Delta_{x} f\right|+\left|\Delta_{y} f\right| \\
& =\left|\begin{array}{l}
(f(i-1, j-1)+2 f(i-1, j)+f(i-1, j+1))- \\
(f(i+1, j-1)+2 f(i+1, j)+f(i+1, j+1))
\end{array}\right| \\
& +\left|\begin{array}{l}
(f(i-1), j-1)+2 f(i, j-1)+f(i+1, j-1))- \\
(f(i-1, j+1)+2 f(i, j+1)+f(i+1, j+1))
\end{array}\right|
\end{aligned}
$$


The convolution operator is as follows:

$\Delta_{x} f=\left|\begin{array}{lll}-1 & 0 & 1 \\ -2 & 0 & 2 \\ -1 & 0 & 1\end{array}\right|, \Delta_{y} f=\left|\begin{array}{ccc}-1 & -2 & -1 \\ 0 & 0 & 0 \\ 1 & 2 & 1\end{array}\right|$

Threshold TH is taken appropriately, and the following judgment is made; for example, $s(i, j)>T H, \quad(i, j)$ is for the step-like edge points, $\{s(i, j)\}$ is for edges of the image.

(2) Because the extreme points are at the edges, the second order derivative is equal to zero. It can take points on either side of the edge points within a small neighborhood points based on this feature, the direction of the gradient value $\theta$ of each point in this small field is calculated. The calculation formula is as follows:

$$
\theta(i, j)=\cot \left(f_{y}^{\prime} / f_{x}^{\prime}\right)
$$

The direction of the gradient value $\theta$ of each point in the selected field is get, and the center pixel gradient value is compared with the two pixel gradient along the gradient direction value. If the gradient magnitude at the center of the neighborhood is larger than the gradient magnitude between two consecutive points along the gradient direction, this point is judged as the edge points, and the assignment is 1 ; otherwise, if the point is the non edge points, the assignment is 0 , so as to get the edge image of single pixel. The image edge obtained by traditional Sobel operators is wide, and the image edges obtain by this method can be single-pixel precision level.

(3) The pixel values of each edge gradient and gradient direction value are obtained by the above two steps, according to the two values to Gauss curve fitting in the gradient direction. Gauss curve expression is as follows:

$y=\frac{1}{\sqrt{2 \pi \sigma}} \exp \left(\frac{-(x-\mu)^{2}}{2 \sigma^{2}}\right)$

Logarithmic respectively on both sides of the formula (3.4):

$\ln y=-\frac{(x-\mu)^{2}}{2 \sigma^{2}}+\ln \frac{1}{\sqrt{2 \pi \sigma}}$

Formula (3.5), is a typical quadratic curve, so the edge points are to fit near to the domain pixel gradient of logarithm value with a binary quadratic polynomial. Curved surface fitting function is get, and the quadratic curve is obtained by curved surface fitting function. Assume that a quadratic surface fitting function is as follows:

$f(x, y)=a_{0}+a_{1} x+a_{2} x^{2}+a_{3} y+a_{4} y^{2}+a_{5} x y$

The $(\mathrm{x}, \mathrm{y})$ is the pixel coordinate value, and $f(x, y)$ is the pixel point gradient magnitude of logarithm value. The gray value of pixels is used near the edge of the $3 \times 3$ field, and the least square method is to calculate the unknown coefficient in fitting function. The solving formula is as follows:

$\delta_{\min }^{2}=\sum_{x=i-1 x=j-1}^{x=i+1 x=j+1}\left[f(x, y)-\left(a_{0}+a_{1} x+a_{2} x^{2}+a_{3} y+a_{4} y^{2}+a_{5} x y\right)\right]^{2}$

The two curved surface function coefficients are obtained from formula (3.7), with the edge point as the center, polar coordinates are established. $(x, y)$ are replaced by $\rho \cos (\theta), \rho \sin (\theta)$ respectively in the curved surface formula (3.6), so as to obtain quadratic curve equation. Because this equation is the gradient direction of change of curvature equation, 
the maximum value is accurate coordinate of the edge points. The following is quadratic curve equation:

$$
\rho=-\frac{a_{1} \cos (\theta)+a_{3} \sin (\theta)}{2\left[a_{2} \cos ^{2}(\theta)+a_{5} \cos (\theta) \sin (\theta)+a_{4} \sin ^{2}(\theta)\right]}
$$

Then the sub pixel edge point coordinate is obtained:

$$
x=\rho_{g} \cos (\theta) \quad y=\rho_{g} \sin (\theta)
$$

The light, belt deflection angle information, is detected by the above sub pixel edge detection operator, and two clip line images are on same computer screen with software design, when the double clamp single line, the system is alignment and have a series value about the measured lens focal length calculation.

\section{Conclusion}

According to the mathematical relationship between telescope magnification and lens focal length to derive, the optical system focal length calculation is realized by measuring the imaging angle on infinite light away from the axis Based on the applied optics light path transmission theory. CCD imaging technology, grating angular displacement measuring technology and digital image processing technology are used in the focal length measurement system in order to achieve automation and intelligent detection optical system focal length. This method is used to duplicate measurement in same precision grade. When the focal length is $10 \mathrm{~mm}$, the accuracy is higher to reach $1.36 \times 10-4$; when the focal length is $1000 \mathrm{~mm}$, the accuracy is lower to reach $5.2 \times 10-4$. The experimental results show that, the precision of the measured optical lens group in the measurement system is higher than the current level of focal length measurement.

\section{References}

[1] Z. Nan, L. Zhenwu and L. Fengyou, "Optical design of diffractive telescope", Infrared and Laser Engineering. vol. 36, no. 1, (2007), pp. 106-108.

[2] M. H. Gao, L. F. Li and J. L. Xu, "Design of a novel multi band of large aperture collimator", Infrared and Laser Engineering, vol. 38, no. 4, (2009), pp. 698-701.

[3] W. Lei, S. Y. Wang and Z. Hu, "The basic principle and method of parallel light pipe”, Chinese Journal of Scientific Instrument, S. 1, (2006), pp. 30-33.

[4] C. Q. Miao, W. Bo and M. Y. Fu, "Research on CCD real-time imaging tracking system", Infrared and Laser Engineering, vol. 34, no. 3, (2005), pp. 310-313.

[5] Y. L. Zhao and Z. L. Xu, "The improvement of micro slit width laser measurement system based on the CCD", Infrared and Laser Engineering, vol. 25, no. 1, (2006), pp. 75-77.

[6] Z. Yu, Z. Y. An and R. N. Yang, "The applications of CCD subdivision in the telescope exit pupil diameter and exit pupil distance measurement”, Infrared and Laser Engineering, vol. 37, no. 5, (2008), pp 894-896.

[7] Y. S. Jiang, "Research on Application of Analog-probe for Measuring Center, Harbin University of Science and Technology”, vol. 14, no. 4, (2009) August, pp.121-124

[8] Q. K. SONG, "I'm prove Fuzzy C - means Clustering Algorithm, Harbin University of Science and Technology”, vol. 12, no. 4, (2007) August.

[9] T. T. BAI, "Image Edge Detection Based on Wavelet Transform and Canny Operator, Harbin University of Science and Technology", vol. 15, no. 1, (2010) Febuary. 